\title{
Review
}

\section{Nutritional status in the pediatric oncology patients}

\author{
Silvia Triarico ${ }^{1}$, Emanuele Rinninella ${ }^{2}$, Giorgio Attinà ${ }^{1}$, Alberto Romano ${ }^{1}$, Palma Maurizi ${ }^{1,3}$, \\ Stefano Mastrangelo ${ }^{1,3}$, Antonio Ruggiero ${ }^{1,3, *}$ \\ ${ }^{1}$ Pediatric Oncology Unit, Fondazione Policlinico Universitario A. Gemelli IRCCS, 00168 Rome, Italy \\ ${ }^{2}$ UOC of Clinical Nutrition, Department of Medical and Surgical Sciences, Fondazione Policlinico Universitario A. Gemelli IRCCS, 00168 Rome, Italy \\ ${ }^{3}$ Università Cattolica Sacro Cuore, 00168 Rome, Italy \\ *Correspondence: antonio.ruggiero@unicatt.it (Antonio Ruggiero) \\ Academic Editor: Qingping Dou \\ Submitted: 16 November 2021 Revised: 28 December 2021 Accepted: 29 December 2021 Published: 8 February 2022
}

\begin{abstract}
Nutritional status plays a vital role in the growth of children. In pediatric patients, disease-related malnutrition is a dynamic and multifactorial process supported by several factors such as inflammation, increased energy expenditure, decreased intake or reduced utilization of nutrients. In pediatric patients with malignancies, sarcopenia may coexist with malnutrition, amplifying its negative impact on prognosis. Careful monitoring of nutritional status both at diagnosis and during chemotherapy treatment allows early detection of the risk and/or presence of malnutrition. A rapid and personalized nutritional intervention can improve adherence to treatment, reduce complications and improve the patients' quality of life.
\end{abstract}

Keywords: Sarcopenia; Pediatric oncology; Malnutrition; Cancer; Children

\section{Malnutrition and sarcopenia in pediatric oncology patients}

In 2013, the Academy of Nutrition and Dietetics and American Society of Parental and Enteral Nutrition (ASPEN) defined pediatric malnutrition as an imbalance in nutrient requirements and intake that causes adverse effects on growth, neurocognitive and body functions, and other relevant clinical outcomes [1]. Malnutrition has long been described in pediatric populations with a high prevalence in children with chronic diseases [2]. The pathogenesis of malnutrition in children with cancer is related to increased energy needs and losses, but also to decreased micro- and macronutrient intake [3-5].

Pro-inflammatory cytokines (TNF-, IL-1, IL-6, IFN) released from the tumor can increase the metabolic and catabolic rate, resulting in mobilization and oxidation of energy substrates and protein loss [6-8]. Similarly, chemotherapy not only increases inflammation due to cytokine secretion, but also common gastrointestinal disturbances due to chemotherapy toxicity (e.g., vomiting, diarrhea, malabsorption, mucosal damage, gastrointestinal infections) may lead to increased energy losses. In addition, chemotherapy drugs can produce changes in taste, loss of appetite, nausea, and/or vomiting, resulting in reduced nutrient intake [9-12]. All these processes lead to neoplastic cachexia, a complex metabolic syndrome characterized by a continuous loss of skeletal muscle mass (with or without loss of fat mass). These processes cannot be completely reversed by conventional nutritional support, leading to progressive functional impairment, with weight loss or inadequate weight gain, anorexia, muscle atrophy, fa- tigue, and abnormal biochemical parameters (low serum albumin, anemia, increased inflammatory markers) [13-15].

Unlike malnutrition, sarcopenia is a new term in the pediatric literature. Sarcopenia has been characterized in adults as a component of malnutrition, described by loss of skeletal muscle mass (SMM) and reduced muscle strength or physical performance [16].

However, in pediatrics, sarcopenia has only recently been identified in the context of reduced SMM. Sarcopenia is a pathological condition, characterized by a progressive and generalized reduction in the quantity, quality and strength of muscle mass, more or less associated with reduced physical performance. It is a major cause of physical disability, poor quality of life, loss of self-sufficiency and death [17]. This condition is typically associated with aging, as muscle mass after the first fifty years declines by approximately $0.5-1 \%$ per year in females and males, respectively [17].

However, there are pathological conditions of systemic inflammation (chronic inflammatory diseases, endocrine diseases, cancer) that determine its occurrence even at a young age, as an effect of the catabolic state of skeletal muscle damage generated by inflammatory cytokines [18].

In pediatric patients with neoplasms, sarcopenia may co-occur in the context of malnutrition, amplifying its negative impact on patient prognosis [19-21]. Malnutritionsarcopenia syndrome, which is the co-occurrence of two clinical conditions, has been reported in the adult literature with considerable overlap in outcomes and adverse interventions [22-24]. 
Similarities between the definitions of sarcopenia and malnutrition include overlapping principles related to lean mass depletion, alterations in muscle function, and suboptimal nutrient intake leading to nutritional deficiencies, fine motor development, and cognition.

There are several mechanisms that may be involved in the onset and progression of sarcopenia. These mechanisms involve protein synthesis, proteolysis, neuromuscular integrity, and muscle fat content, among others. Recognizing these mechanisms and their underlying causes should facilitate the design of intervention trials targeting one or more of the underlying mechanisms. In some individuals, a clear, single cause of sarcopenia can be identified. In other cases, no obvious cause can be isolated. Therefore, the categories of primary sarcopenia and secondary sarcopenia may be useful in clinical practice. Sarcopenia may be considered "primary" (or age-related) when no cause other than aging itself is evident, whereas sarcopenia may be considered "secondary" when one or more other causes are evident. In many older people, the aetiology of sarcopenia is multifactorial, so it may not be possible to characterize every individual as having a primary or secondary condition [17].

The European Working Group on Sarcopenia in Older People (EWGSOP) suggests the distinction between "presarcopenia", "sarcopenia" and "severe sarcopenia". The "presarcopenia" phase is characterized by low muscle mass without impact on muscle strength or physical performance. This phase can only be identified by techniques that measure muscle mass accurately and with reference to standard populations. The "sarcopenia" stage is characterized by low muscle mass in addition to low muscle strength or physical performance. "Severe sarcopenia" is the stage identified when all three criteria of the definition are met (low muscle mass, low muscle strength, and low physical performance). Recognizing the stages of sarcopenia can aid in selecting treatments and setting appropriate recovery goals [17].

EWGSOP2 recently identifies the subcategories of sarcopenia as acute and chronic. Sarcopenia that has lasted less than 6 months is considered an acute condition, while sarcopenia that lasts $\geq 6$ months is considered a chronic condition. Acute sarcopenia is usually related to an acute disease or injury, whereas chronic sarcopenia is likely to be associated with chronic and progressive conditions and increases the risk of mortality. This distinction is intended to emphasize the need to conduct periodic assessments of sarcopenia in individuals who may be at risk for sarcopenia in order to determine how quickly the condition is developing or worsening. Such observations are expected to facilitate early intervention with treatments that may help prevent or delay the progression of sarcopenia and its consequences [17].

Malnutrition may adversely affect overall survival because it may reduce tolerance to chemotherapy, increase treatment-related mortality, and decrease disease-free sur- vival [25-29]. In addition, it may reduce the tolerated and absorbed dose of chemotherapeutics, resulting in reduced response to treatment [30-33].

Disease-related malnutrition has been associated with decreased immunity and increased risk of infection and febrile neutropenia (FN) due to hormonal changes and impaired cytokine response. When a patient has febrile neutropenia, it is mandatory to hospitalize the patient to perform intravenous antibiotic therapy. As a result, the number of hospitalizations in malnourished children and adolescents with cancer become significantly higher, producing increased costs and worsening quality of life (QoL) [34].

In hospitalized children, early and appropriate nutritional intervention is helpful in preventing stunting, promoting therapeutic tolerance, improving quality of life, and reducing the length of hospital stay [35,36]. ESPEN and the European Society for Pediatric Gastroenterology, Hepatology and Nutrition (ESPGHAN) recommend nutritional risk screening at the time of hospital admission to aid in the identification of at-risk children and allow implementation of an appropriate supportive nutrition plan [37]. Although several pediatric nutritional risk scores are reported in the literature, there is no consensus on an "ideal" screening tool and, often, nutritional screening is still not widely performed [38].

By definition, a nutritional screening tool should not be time-consuming, should be simple to perform in daily practice, easily understandable, sensitive and specific, and applicable and reliable for a broad group of diseases [39].

To date, seven main screening tools for nutritional risk are available for children: Nutrition Risk Score (NRS), Pediatric Nutrition Risk Score (PNRS), Subjective Global Nutritional Assessment (SGNA), Screening Tool for the Assessment of Malnutrition in Pediatrics (STAMP), Paediatric Yorkhill Malnutrition Score (PYMS), Screening Tool for Risk on Nutritional status and Growth (STRONGkids), and Paediatric Nutrition Screening Tool (PNST) [36,40,41]. In 2015, Huysentruyt et al. [42] performed a meta-analysis including 11 studies that included at least one score among PNRS, STAMP, PYMS, and STRONGkids. The authors concluded that there is no single screening means that is preferable to others because each should be used in a specific setting. For example, STRONGkids is the best option in all age groups as a rapid screening means, PYMS or STAMP may be preferred if anthropometric measurements are needed at the time of hospital admission.

After the nutritional risk assessment, which is necessary for all hospitalized patients, a percentage of patients may be at risk of malnutrition. In this case, a specific nutritional assessment is mandatory. An accurate medical and dietary history must be collected. The anamnesis should include the evaluation of the growth curve, the possible onset of puberty and psychomotor development with feeding capacity. Any prior acute and chronic illnesses, hospitalizations, and surgical procedures should be sought, with spe- 
cial attention to nutrition-related diseases. It is important to establish the duration of the current illness, documenting motor and swallowing abilities, gastrointestinal symptoms, weight changes. The use of certain medications, which may cause nutritional deficiency, the use of vitamins, minerals, herbal products and supplements should be reported for possible drug interactions. The number of meals, eating habits, food allergies and intolerances, are assessed retrospectively ( $24 \mathrm{~h}$ dietary recall) or prospectively (nutritional diary, usually 3 to 7 days) [43].

In addition, the Food Frequency Questionnaire (FFQ) gives more information on eating habits allowing to evaluate a possible correlation between diet and pathological condition of the child.

The objective examination evaluates the patient's general condition and investigates the presence of signs of specific nutritional deficiencies. In particular, abnormal findings in the examination of hair, eyes, lips and mouth, tongue, teeth, skin, nails, are usually related to specific micronutrient deficiencies (e.g., zinc, iron, essential fatty acids, selenium, magnesium, vitamin $\mathrm{A}$, vitamin $\mathrm{C}, \mathrm{B} 12$, folate). Inspection may reveal protein malnutrition by the presence of limb edema, distended abdomen, and muscle atrophy.

Laboratory data play a complementary role in assessing nutritional status, although no single laboratory test can provide a complete assessment of nutritional status. Disease-related malnutrition is often associated with an inflammatory state that promotes a catabolic effect on free fat body mass and muscle protein. The presence of inflammation should be identified because it can decrease the effectiveness of nutritional intervention. Acute phase proteins (C-reactive protein, fibrinogen, haptoglobin, ceruloplasmin, ferritin, and alpha-1-antitrypsin) have high levels during the acute inflammation or catabolic state; in contrast, albumin, prealbumin, retinol-binding protein (RBP), and transferrin are decreased in these cases [44].

Fig. 1 summarizes the pathogenetic mechanisms of sarcopenia in children with cancerresume.

\section{Anthropometric variables}

Weight is a measure of overall nutritional status, but can be influenced by many variables such as age, gender, daily intake, and hydration status. Patients over 2 years of age and able to stand should be weighed on a platform scale with moving weights or digital scale. For patients unable to stand, bed scales or wheelchair scales are used. Children under two years of age should be weighed in the supine position in a scale, ensuring that the weight is distributed equally on each side of the center of the scale.

Stature (length, height or alternative measures) is a very important measure for observing long-term nutritional status. For children under 2 years of age, length is obtained using a supine infantometer. For children over 2 years of age, an upright stadiometer is used, if possible fixed to the wall.

Head circumference can be obtained in children up to 36 months of age, using a flexible tape measure placed around the head. Head circumference should be considered an index of brain development and nutritional status.

Weight-for-height can be used as an evaluation parameter in children who are less than 2 years old; it corresponds to the BMI used in patients over 2 years old. BMI is calculated using the following formula BMI = Weight $(\mathrm{kg}) /$ Height $\left(\mathrm{m}^{2}\right)$. Given the variability in sex and age, several specific BMI values are available for children [45].

In paediatric age, BMI can be used to identify a condition of overweight or obesity. Although the World Health Organization (WHO) has validated the use of BMI also to assess thinness in adolescence, it is not used to identify an underweight condition in children: in fact, in children, underweight is expressed as "low weight for age". In addition, BMI should not be used as the sole indicator of nutritional status in children with clinical conditions because BMI does not consider differences in body composition.

Mid-Upper-Arm-Circumference (MUAC) is a simple measurement taken from a flexible tape placed perpendicular to the long axis of the arm, which is flexed at $90^{\circ}$. The midpoint of the upper arm midway between the acromion and olecranon is measured and marked. MUAC is a better indicator of body composition than BMI in patients with edema because it is not affected by hydration status.

The thickness of the triceps skin fold is measured by grasping the skin and subcutaneous adipose tissue between the thumb and forefinger above the point already identified for MUAC. This is a parameter that is often used for research, but can also be useful in identifying deposits of body fat in the patient.

The handgrip test is a force measurement performed using a handheld dynamometer, which is a non-invasive, low-cost tool for measuring functional muscle status. Using the dynamometer, the patient performs a sequence of movements that mimics the maximum strength of the hand and forearm muscles. Nutritional changes affect muscle function sooner than muscle mass, so grip strength can help detect the presence of malnutrition in children. Age and sex percentiles express the position of a child's measurement (weight, length or height, weight-for-length or BMI) on a standard bell-shaped reference curve derived from population data. A percentile indicates the percentage of the population that remains above or below that measured in the child, helping to compare the child's position with a population of other children similar in age and sex. However, according to WHO statements, percentiles do not accurately indicate the patient's actual degree of deviation from population standards; instead, the use of Z-scores would be better for expressing anthropometric measures [1].

$\mathrm{Z}$-scores are more sensitive than percentiles because they express in standard deviation (SD) how far the child is from the mean by comparing individual anthropometric 


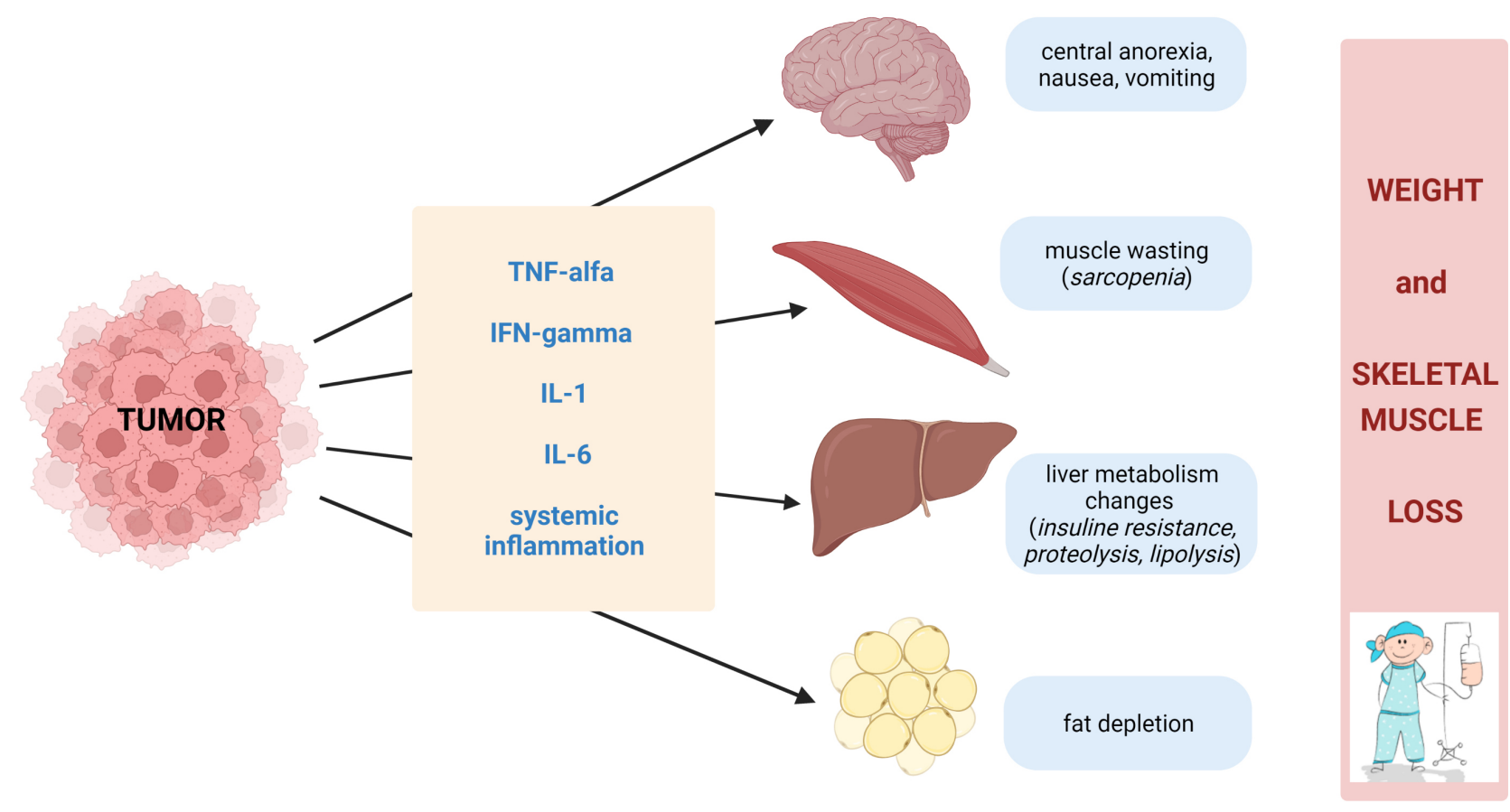

Fig. 1. Pathogenesis of sarcopenia by the tumor. Cancer cells produce TNF-alfa, IFN-gamma, IL-1, IL-6 causing a chronic inflammatory state which in turn determines fat depletion, liver metabolism changes, muscle wasting with the appereance of weight and skeletal muscle loss.

measurement with data from the reference age groups. The source of material used and relevant ethical framework for all experiments should be clearly identified (ethics approval and/or written informed consent). Methods already published should be indicated by a reference: only relevant modifications should be described. This implies that a full description of all the experiments described in Results and presented in the Figures/Tables is expected in this section. For each experiment, all steps need to be mentioned, along with instruments the analyses were performed on, reagents and methods to permit the replication of the work by others. We would encourage authors to submitting a detailed Bio-protocol.

\section{Body composition methods}

There are a number of techniques for routine determination of coporeal composition, e.g., Total Body Potassium Counting (TBK), Dual-energy X-ray absorptiometry (DXA), Bio impedance (BIA). Although these reference methods are routinely used, each has inherent practical limitations [46].

Dual-energy X-ray Absorptiometry (DXA) is a noninvasive method that can be applied to patients of all ages to measure the composition of body regions. It is a rapid, lowcost technique that involves low radiation exposure [47].

Bio impedance analysis (BIA) is another safe, noninvasive and easily implemented method used for indirect determination of body composition. It is based on the prin- ciple that the conduction of an alternating electric current in a body can find a resistance to passage (impedance) inversely proportional to the content of water and electrolytes. Free-fat mass (made up of well-hydrated cells) has a relatively slow impedance, while Fat mass (low in water and electrolytes) opposes a high impedance. Bones, air (in the lungs) and parenchymal organs are not considered good conductors and are not taken into account. The impedance (Z) to the passage of current through the body consists of two components: resistance $(\mathrm{R})$ and reactance $(\mathrm{Xc})$. $\mathrm{R}$ is essentially dependent on extracellular water (ECW) and FM. $\mathrm{Xc}$ is an indirect measure of the body's cell mass: it is the quality of healthy cell membranes to take an electrical charge and release it at a later time.

\section{Analysis of sarcopenia}

Early detection of sarcopenic status may allow for the timely application of appropriate and tailored nutritional interventions to correct it. There are several ways to assess muscle mass and possible sarcopenia status in clinical practice $[48,49]$.

Weight, body mass index, mid-arm circumference, and skinfold thickness of the triceps are anthropometric values that are easily influenced by the disease state or concomitant therapies and do not provide a precise assessment of body composition [50].

In contrast, bone densitometry (DXA), bioelectrical impedance analysis (BIA), and air displacement plethys- 
mography (ADP) are reliable body composition analysis techniques, but they require dedicated personnel and equipment, thus limiting their wider use [51,52].

Imaging modalities (CT and MRI) are the gold standard in quantifying SMM in adults [16]. High radiation exposure is the main disadvantage of the routine use of CT for the assessment of sarcopenia in pediatric populations. Although MRI is radiation-free, it is more expensive than CT, which may limit its use in serial measurements [53].

In children with chronic diseases, imaging is often performed as part of their routine clinical care, making it possible to assess SMM without additional cost and radiation exposure. A recent study in adults demonstrated that total body fluid status can influence the determination of SMM derived from cross-sectional CT/MRI [51,54]. This may be applicable to pediatric clinical populations that are prone to altered total body fluid status and thus warrant further study.

An important consideration in the assessment of body composition in pediatrics includes careful evaluation of overall growth, which may affect the assessment of sarcopenia in children. While female and male children may have comparable fat mass and lean mass during early childhood, important changes in relative SMM and fat mass occur during the pubertal growth phase. During puberty, it has been reported that females gain more fat mass, whereas males have a greater increase in lean mass triggered by endocrine factors, particularly growth hormones and sex hormones (oestrogen, testosterone) [55].

Currently, there is no gold standard tool for assessing motor function impairment in infants evaluated for sarcopenia [56]. It is difficult to assess muscle function in a standardized manner with infants, as their motor performance is determined by a variety of factors, including the development of postural control, coordination, core stability, and ability to perform focused, isolated movements. In addition, the presence of disease may also limit the ability to perform specific muscle tests. Within the emerging literature on sarcopenia in children, motor function assessments have not yet been applied to the assessment of sarcopenia in early childhood. In older children (school-aged children/adolescents), the handgrip test and the 6-minute walk test are well documented for determining upper body strength and functional exercise performance, respectively $[57,58]$.

These tests are consistent with recommended tests to assess potential deficits in muscle function within adult definitions of sarcopenia. Although other muscle strength tests (e.g., push-ups, pull-ups, and standing broad jump) and performance tests (stair climb test, sit-to-stand test) have been used in the pediatric population, they are constrained by the absence of standardized protocols [57,59-62].

Measurement of psoas muscle area (SMA) from single-section abdominal computed tomography (CT) images at L3-L4 and L4-L5 is an easily accessible, rapid, and reliable method of assessing muscle mass. By relating the SMA $\left(\mathrm{cm}^{2}\right)$ to the square of the patient's height $\left(\mathrm{m}^{2}\right)$, the muscle mass index (SMI) is calculated, which is indicative of sarcopenia if less than $55 \mathrm{~cm}^{2} / \mathrm{m}^{2}$ in males and 39 $\mathrm{cm}^{2} / \mathrm{m}^{2}$ in females $[63,64]$. This method has been widely adopted for the analysis of muscle mass in adult patients with neoplasia, routinely subjected to axial tomography examinations of disease reassessment, as well as in patients with liver disease, inpatients in intensive care, and patients undergoing surgery [17,65-71].

This method has been widely used for the diagnosis of sarcopenia secondary to chronic disease (oncological pathologies, inflammatory bowel disease, type 2 diabetes, end-stage liver disease, intestinal insufficiency) also in pediatric patients [72-77].

Recently, Lurz et al. [78] generated age- and sexspecific curves related to total psoas muscle area (tPMA) obtained at L3-L4 or L4-L5, which can be used for pediatric patients aged 1-16 years. From these, the Z-Scores of the PMA can be obtained through a calculator available online [https://ahrc-apps.shinyapps.io/sarcopenia/] and easily identify the presence of sarcopenia.

Although in their study, Lurz et al. [78] analyze tPMA at both the L3-L4 and L4-L5 levels, tPMA at the L4-L5 level appears to be more relevant in children aged 1-16 years, as at this level the shape of the psoas muscle is also rounder than at L3-L4, allowing for more accurate contour drawing. In addition, L4-L5 is the reference level for the assessment of visceral adipose tissue, so an analysis at this level is a reliable measure of both skeletal muscle and adipose tissue [79-85].

\section{Discussion}

In presence of a high risk of malnutrition, it is necessary to perform a comprehensive nutritional assessment that must involve the pediatrician and clinical nutritionist. Medical and dietary history, physical examination, and anthropometric measurements should be evaluated for this purpose.

The increase of survival in subjects suffering from neoplastic pathology also in pediatric age poses the problem of the type of life of these subjects and of the interventions to promote in the best way situations of well-being. In this sense, in the light of the increase of pathologies related to the development of atherosclerotic disease, it is necessary to prepare all those studies and strategies useful to define the most appropriate interventions to prevent these diseases. If on the one hand the possible role of the established therapy (radiation or chemotherapy) poses the problem of specific experimental studies, the concomitant presence of poor dietary habits and sedentary lifestyles bring to the forefront the need for preventive interventions on these factors. At present, a pathway aimed at wellness in the subject recovered from a neoplastic process in pediatric age must perform an adequate nutritional assessment, evaluate the eating habits (through recall 24 hours and dietary diary) of these 
subjects, propose specific courses of correct eating habits and practice of daily spontaneous movement. Pharmacokinetic interactions between food and chemotherapies must be evaluated for each individual chemotherapeutic agent, as these interactions are complex and not generalizable [8688]. Therefore, the potential interference of food with the pharmacokinetic parameters of the drug should always be kept in mind when prescribing an oral chemotherapeutic drug. These interferences can produce changes in bioavailability that, in the case of drugs with a narrow therapeutic index, lead to an increased risk of therapeutic failure or, conversely, to an increase in undesirable effects.

Metabolic-nutritional support should be initiated as early as possible after diagnosis and pursued continuously in order to prevent or correct in a timely manner the alterations in nutritional status induced by the underlying disease and radiochemotherapy treatments. Today it is increasingly clear that the metabolic-nutritional therapy for the cancer patient can only be based on a combined and multimodal approach, e.g., conventional nutritional therapy combined with drugs or nutrients able to modulate the inflammatory response.

Dietary education should take place during the patient's hospitalization, in order to sensitize both him and his family to adopt a dietary pattern appropriate to the situation [89].

In the discharge phase the patient, or his family, must be instructed to follow an appropriate dietary regimen, through the help and support of easy and understandable dietary patterns (foods to prefer/limit/avoid) or personalized diets. For this reason it is essential to include in the therapeutic program of the oncological pathology also the nutritional treatment, which must have these objectives: nutritional support must be personalized, keeping in mind the prognosis of the underlying pathology, so as to adapt the intensity of dietary intervention; promote and stimulate the diet by os, avoiding "closed" diets; sensitize the patient and his family to proper nutrition, both during hospitalization and at discharge; articulate a periodic follow-up.

Metabolic-nutritional intervention in oncology must now be considered as an integral part of care and started from the beginning of the course of the disease: the sooner this "parallel path" starts, integrated with cancer therapy and highly codified and individualized, the higher the chances are to contain or slow down the loss of body weight. Based on the increasing knowledge of the mechanisms that lead to weight loss and thanks to the increased availability of metabolically active nutritional substrates, the metabolic-nutritional support in oncology will undergo significant changes in the coming years, offering the oncologist a valid and irreplaceable tool to make cancer therapy more effective.

\section{Conclusions}

There are several ways to assess muscle mass and the possible state of sarcopenia in clinical practice. Weight, body mass index, mid-arm circumference, and triceps skinfold thickness are anthropometric values that are easily influenced by disease status or concomitant therapies and do not provide a precise assessment of body composition. Among the available tools, the measurement of the psoas muscle area, derived from single cross-sectional abdominal computed tomography images at the level of L3-L4 and L4-L5 represents an easily accessible, rapid and reliable method to assess muscle mass. Early detection of the sarcopenic state may allow timely application of appropriate and personalized nutritional interventions to correct it.

\section{Author contributions}

ST and ER designed the research study. GA and ARo performed the research. SM analyzed the data. ST, ARu and PM wrote the manuscript. All authors contributed to editorial changes in the manuscript. All authors read and approved the final manuscript.

\section{Ethics approval and consent to participate}

Not applicable.

\section{Acknowledgment}

The authors thank the 'Sara un angelo con la bandana onlus' Association for their support to the paediatric research.

\section{Funding}

This research received no external funding.

\section{Conflict of interest}

The authors declare no conflict of interest.

\section{References}

[1] Mehta NM, Corkins MR, Lyman B, Malone A, Goday PS, Carney $\mathrm{LN}$, et al. Defining pediatric malnutrition: a paradigm shift toward etiology-related definitions. Journal of Parenteral and Enteral Nutrition. 2013; 37: 460-481.

[2] Joosten KFM, Hulst JM. Prevalence of malnutrition in pediatric hospital patients. Current Opinion in Pediatrics. 2008; 20: 590 596.

[3] Sala A, Pencharz P, Barr RD. Children, cancer, and nutrition-a dynamic triangle in review. Cancer. 2004; 100: 677-687.

[4] Ladas EJ, Sacks N, Meacham L, Henry D, Enriquez L, Lowry G, et al. A Multidisciplinary Review of Nutrition Considerations in the Pediatric Oncology Population: a Perspective from Children's Oncology Group. Nutrition in Clinical Practice. 2005; 20: 377-393.

[5] Iniesta RR, Paciarotti I, Brougham MFH, McKenzie JM, Wilson DC. Effects of pediatric cancer and its treatment on nutritional status: a systematic review. Nutrition Reviews. 2015; 73: 276295.

[6] Skipworth RJE, Stewart GD, Dejong CHC, Preston T, Fearon 
$\mathrm{KCH}$. Pathophysiology of cancer cachexia: much more than host-tumour interaction? Clinical Nutrition. 2007; 26: 667-676.

[7] Mignini EV, Scarpellini E, Rinninella E, Lattanzi E, Valeri MV, Clementi $\mathrm{N}$, et al. Impact of patients nutritional status on major surgery outcome. European Review for Medical and Pharmacological Sciences. 2018; 22: 3524-3533.

[8] Picton SV. Aspects of altered metabolism in children with cancer. International Journal of Cancer. 1998; 78: 62-64.

[9] Brinksma A, Huizinga G, Sulkers E, Kamps W, Roodbol P, Tissing W. Malnutrition in childhood cancer patients: a review on its prevalence and possible causes. Critical Reviews in Oncology/Hematology. 2012; 83: 249-275.

[10] Ruggiero A, Rizzo D, Trombatore G, Maurizi P, Riccardi R. The ability of mannitol to decrease cisplatin-induced nephrotoxicity in children: real or not? Cancer Chemotherapy and Pharmacology. 2016; 77: 19-26.

[11] Rinninella E, Annetta MG, Serricchio ML, Dal Lago AA, Miggiano GAD, Mele MC. Nutritional support in acute pancreatitis: from physiopathology to practice. an evidence-based approach. European Review for Medical and Pharmacological Sciences. 2017; 21: 421-432.

[12] Ruggiero A, Rizzo D, Catalano M, Coccia P, Triarico S, Attiná G. Acute chemotherapy-induced nausea and vomiting in children with cancer: still waiting for a common consensus on treatment. The Journal of International Medical Research. 2018; 46: 2149-2156.

[13] Fearon K, Strasser F, Anker SD, Bosaeus I, Bruera E, Fainsinger RL, et al. Definition and classification of cancer cachexia: an international consensus. The Lancet. Oncology. 2011; 12: 489495.

[14] Evans WJ, Morley JE, Argilés J, Bales C, Baracos V, Guttridge D, et al. Cachexia: a new definition. Clinical Nutrition. 2008; 27: 793-799.

[15] Co-Reyes E, Li R, Huh W, Chandra J. Malnutrition and obesity in pediatric oncology patients: causes, consequences, and interventions. Pediatric Blood \& Cancer. 2012; 59: 1160-1167.

[16] Cruz-Jentoft AJ, Baeyens JP, Bauer JM, Boirie Y, Cederholm T, Landi F, et al. Sarcopenia: European consensus on definition and diagnosis: Report of the European Working Group on Sarcopenia in Older People. Age and Ageing. 2010; 39: 412-423.

[17] Cruz-Jentoft AJ, Bahat G, Bauer J, Boirie Y, Bruyère O, Cederholm T, et al. Sarcopenia: revised European consensus on definition and diagnosis. Age and Ageing. 2019; 48: 601-601.

[18] Mitchell WK, Williams J, Atherton P, Larvin M, Lund J, Narici M. Sarcopenia, dynapenia, and the impact of advancing age on human skeletal muscle size and strength; a quantitative review. Frontiers in Physiology. 2012; 3: 260.

[19] Colloca G, Di Capua B, Bellieni A, Cesari M, Marzetti E, Valentini $\mathrm{V}$, et al. Muscoloskeletal aging, sarcopenia and cancer. Journal of Geriatric Oncology. 2019; 10: 504-509.

[20] Falsini B, Iarossi G, Chiaretti A, Ruggiero A, Manni L, Luigi $\mathrm{M}$, et al. NGF eye-drops topical administration in patients with retinitis pigmentosa, a pilot study. Journal of Translational Medicine. 2016; 14: 8.

[21] Ruggiero A, Triarico S, Trombatore G, Battista A, Dell'acqua F, Rizzari C, et al. Incidence, clinical features and management of hypersensitivity reactions to chemotherapeutic drugs in children with cancer. European Journal of Clinical Pharmacology. 2013; 69: 1739-1746.

[22] Juby AG, Mager DR. A review of nutrition screening tools used to assess the malnutrition-sarcopenia syndrome (MSS) in the older adult. Clinical Nutrition ESPEN. 2019; 32: 8-15.

[23] Ferrara P, Marrone G, Emmanuele V, Nicoletti A, Mastrangelo A, Tiberi E, et al. Homotoxicological remedies versus desmopressin versus placebo in the treatment of enuresis: a randomised, double-blind, controlled trial. Pediatric Nephrology.
2008; 23: 269-274.

[24] Timeus F, Crescenzio N, Longoni D, Doria A, Foglia L, Pagliano $\mathrm{S}$, et al. Paroxysmal nocturnal hemoglobinuria clones in children with acquired aplastic anemia: a multicentre study. PLoS ONE. 2014; 9: e101948.

[25] Loeffen EAH, Brinksma A, Miedema KGE, de Bock GH, Tissing WJE. Clinical implications of malnutrition in childhood cancer patients-infections and mortality. Supportive Care in Cancer. 2015; 23: 143-150.

[26] Pribnow AK, Ortiz R, Báez LF, Mendieta L, Luna-Fineman S. Effects of malnutrition on treatment-related morbidity and survival of children with cancer in Nicaragua. Pediatric Blood \& Cancer. 2017; 64.

[27] Norman K, Pichard C, Lochs H, Pirlich M. Prognostic impact of disease-related malnutrition. Clinical Nutrition. 2008; 27: 5-15.

[28] Rogers PCJ. Nutritional status as a prognostic indicator for pediatric malignancies. Journal of Clinical Oncology. 2014; 32: $1293-1294$.

[29] Triarico S, Rinninella E, Cintoni M, Capozza MA, Mastrangelo $\mathrm{S}$, Mele MC, et al. Impact of malnutrition on survival and infections among pediatric patients with cancer: a retrospective study. European Review for Medical and Pharmacological Sciences. 2019; 23: 1165-1175.

[30] Sala A, Rossi E, Antillon F, Molina AL, de Maselli T, Bonilla $\mathrm{M}$, et al. Nutritional status at diagnosis is related to clinical outcomes in children and adolescents with cancer: a perspective from Central America. European Journal of Cancer. 2012; 48: 243-252.

[31] Falsini B, Ziccardi L, Lazzareschi I, Ruggiero A, Placentino L, Dickmann A, et al. Longitudinal assessment of childhood optic gliomas: relationship between flicker visual evoked potentials and magnetic resonance imaging findings. Journal of NeuroOncology. 2008; 88: 87-96.

[32] Murry DJ, Riva L, Poplack DG. Impact of nutrition on pharmacokinetics of anti-neoplastic agents. International Journal of Cancer. Supplement. 1998; 11: 48-51.

[33] Triarico $S$, Maurizi $P$, Mastrangelo $S$, Attinà $G$, Capozza MA, Ruggiero A. Improving the Brain Delivery of Chemotherapeutic Drugs in Childhood Brain Tumors. Cancers. 2019; 11: 824.

[34] Brinksma A, Sanderman R, Roodbol PF, Sulkers E, Burgerhof JGM, de Bont ESJM, et al. Malnutrition is associated with worse health-related quality of life in children with cancer. Supportive Care in Cancer. 2015; 23: 3043-3052.

[35] Motil KJ. Sensitive measures of nutritional status in children in hospital and in the field. International Journal of Cancer. Supplement. 1998; 11: 2-9.

[36] Hartman C, Shamir R, Hecht C, Koletzko B. Malnutrition screening tools for hospitalized children. Current Opinion in Clinical Nutrition and Metabolic Care. 2012; 15: 303-309.

[37] Agostoni C, Axelson I, Colomb V, Goulet O, Koletzko B, Michaelsen KF, et al. The need for nutrition support teams in pediatric units: a commentary by the ESPGHAN committee on nutrition. Journal of Pediatric Gastroenterology and Nutrition. 2005; 41: 8-11.

[38] Teixeira AF, Viana KDAL. Nutritional screening in hospitalized pediatric patients: a systematic review. Jornal De Pediatria. 2016; 92: 343-352.

[39] Joosten KFM, Hulst JM. Nutritional screening tools for hospitalized children: methodological considerations. Clinical Nutrition. 2014; 33: 1-5.

[40] Erkan T. Methods to evaluate the nutrition risk in hospitalized patients. Turk Pediatri Arsivi. 2014; 49: 276-281.

[41] Rinninella E, Ruggiero A, Maurizi P, Triarico S, Cintoni M, Mele MC. Clinical tools to assess nutritional risk and malnutrition in hospitalized children and adolescents. European Review for Medical and Pharmacological Sciences. 2017; 21: 2690 
2701.

[42] Huysentruyt K, Devreker T, Dejonckheere J, De Schepper J, Vandenplas Y, Cools F. Accuracy of Nutritional Screening Tools in Assessing the Risk of Undernutrition in Hospitalized Children. Journal of Pediatric Gastroenterology and Nutrition. 2015; 61: 159-166.

[43] Persson LA, Carlgren G. Measuring children's diets: evaluation of dietary assessment techniques in infancy and childhood. International Journal of Epidemiology. 1984; 13: 506-517.

[44] Jensen GL. Inflammation as the key interface of the medical and nutrition universes: a provocative examination of the future of clinical nutrition and medicine. Journal of Parenteral and Enteral Nutrition. 2006; 30: 453-463.

[45] Green Corkins K, Teague EE. Pediatric Nutrition Assessment. Nutrition in Clinical Practice. 2017; 32: 40-51.

[46] Lazzer S, Bedogni G, Agosti F, De Col A, Mornati D, Sartorio A. Comparison of dual-energy X-ray absorptiometry, air displacement plethysmography and bioelectrical impedance analysis for the assessment of body composition in severely obese Caucasian children and adolescents. The British Journal of Nutrition. 2008; 100: 918-924.

[47] Lee SY, Gallagher D. Assessment methods in human body composition. Current Opinion in Clinical Nutrition and Metabolic Care. 2008; 11: 566-572.

[48] Ooi PH, Thompson-Hodgetts S, Pritchard-Wiart L, Gilmour SM, Mager DR. Pediatric Sarcopenia: a Paradigm in the Overall Definition of Malnutrition in Children? Journal of Parenteral and Enteral Nutrition. 2020; 44: 407-418.

[49] Carvalho do Nascimento PR, Poitras S, Bilodeau M. How do we define and measure sarcopenia? Protocol for a systematic review. Systematic Reviews. 2018; 7: 51.

[50] Abd Aziz NAS, Teng NIMF, Abdul Hamid MR, Ismail NH. Assessing the nutritional status of hospitalized elderly. Clinical Interventions in Aging. 2017; 12: 1615-1625.

[51] Cooper C, Fielding R, Visser M, van Loon LJ, Rolland Y, Orwoll $\mathrm{E}$, et al. Tools in the assessment of sarcopenia. Calcified Tissue International. 2013; 93: 201-210.

[52] Orsso CE, Tibaes JRB, Oliveira CLP, Rubin DA, Field CJ, Heymsfield SB, et al. Low muscle mass and strength in pediatrics patients: why should we care? Clinical Nutrition. 2019; 38: 2002-2015.

[53] Sottier D, Petit J, Guiu S, Hamza S, Benhamiche H, Hillon P, et al. Quantification of the visceral and subcutaneous fat by computed tomography: interobserver correlation of a single slice technique. Diagnostic and Interventional Imaging. 2013; 94 : 879-884.

[54] Coccia $P$, Ruggiero A, Rufini V, Maurizi P, Attinà G, Marano $\mathrm{R}$, et al. Cardiac metastases of Ewing sarcoma detected by 18F-FDG PET/CT. Journal of Pediatric Hematology/Oncology. 2012; 34: 236-238.

[55] Loomba-Albrecht LA, Styne DM. Effect of puberty on body composition. Current Opinion in Endocrinology, Diabetes and Obesity. 2009; 16: 10-15.

[56] Griffiths A, Toovey R, Morgan PE, Spittle AJ. Psychometric properties of gross motor assessment tools for children: a systematic review. BMJ Open. 2018; 8: e021734.

[57] Bianco A, Jemni M, Thomas E, Patti A, Paoli A, Ramos Roque J, et al. A systematic review to determine reliability and usefulness of the field-based test batteries for the assessment of physical fitness in adolescents - the ASSO Project. International Journal of Occupational Medicine and Environmental Health. 2015; 28 : 445-478.

[58] Geiger R, Strasak A, Treml B, Gasser K, Kleinsasser A, Fischer $\mathrm{V}$, et al. Six-minute walk test in children and adolescents. The Journal of Pediatrics. 2007; 150: 395-392.

[59] Fernandez-Santos JR, Ruiz JR, Cohen DD, Gonzalez-
Montesinos JL, Castro-Piñero J. Reliability and Validity of Tests to Assess Lower-Body Muscular Power in Children. Journal of Strength and Conditioning Research. 2015; 29: $2277-2285$.

[60] Nunez-Gaunaurd A, Moore JG, Roach KE, Miller TL, KirkSanchez NJ. Motor proficiency, strength, endurance, and physical activity among middle school children who are healthy, overweight, and obese. Pediatric Physical Therapy. 2013; 25: 130-8; discussion 139.

[61] Silva PFS, Quintino LF, Franco J, Faria CDCM. Measurement properties and feasibility of clinical tests to assess sit-tostand/stand-to-sit tasks in subjects with neurological disease: a systematic review. Brazilian Journal of Physical Therapy. 2014; 18: 99-110.

[62] Zaino CA, Marchese VG, Westcott SL. Timed up and down stairs test: preliminary reliability and validity of a new measure of functional mobility. Pediatric Physical Therapy. 2004; 16: $90-98$

[63] Amini B, Boyle SP, Boutin RD, Lenchik L. Approaches to Assessment of Muscle Mass and Myosteatosis on Computed Tomography: a Systematic Review. The Journals of Gerontology: Series a. 2019; 74: 1671-1678.

[64] Anjanappa M, Corden M, Green A, Roberts D, Hoskin P, McWilliam A, et al. Sarcopenia in cancer: Risking more than muscle loss. Technical Innovations \& Patient Support in Radiation Oncology. 2020; 16: 50-57.

[65] van Vugt JLA, Levolger S, de Bruin RWF, van Rosmalen J, Metselaar HJ, IJzermans JNM. Systematic Review and MetaAnalysis of the Impact of Computed Tomography-Assessed Skeletal Muscle Mass on Outcome in Patients Awaiting or Undergoing Liver Transplantation. American Journal of Transplantation. 2016; 16: 2277-2292.

[66] Giusto M, Lattanzi B, Albanese C, Galtieri A, Farcomeni A, Giannelli $\mathrm{V}$, et al. Sarcopenia in liver cirrhosis: the role of computed tomography scan for the assessment of muscle mass compared with dual-energy X-ray absorptiometry and anthropometry. European Journal of Gastroenterology \& Hepatology. 2015; 27: 328-334.

[67] Yeh DD, Ortiz-Reyes LA, Quraishi SA, Chokengarmwong N, Avery L, Kaafarani HMA, et al. Early nutritional inadequacy is associated with psoas muscle deterioration and worse clinical outcomes in critically ill surgical patients. Journal of Critical Care. 2018; 45: 7-13.

[68] Akahoshi T, Yasuda M, Momii K, Kubota K, Shono Y, Kaku $\mathrm{N}$, et al. Sarcopenia is a predictive factor for prolonged intensive care unit stays in high-energy blunt trauma patients. Acute Medicine \& Surgery. 2016; 3: 326-331.

[69] Moisey LL, Mourtzakis M, Cotton BA, Premji T, Heyland DK, Wade CE, et al. Skeletal muscle predicts ventilator-free days, ICU-free days, and mortality in elderly ICU patients. Critical Care. 2013; 17: R206.

[70] Ruggiero A, Lanni V, Librizzi A, Maurizi P, Attinà G, Mastrangelo S, et al. Diagnostic Accuracy of 18F-FDG PET/CT in the Staging and Assessment of Response to Chemotherapy in Children with Ewing Sarcoma. Journal of Pediatric Hematology/Oncology. 2018; 40: 277-284.

[71] Di Cataldo A, Agodi A, Balaguer J, Garaventa A, Barchitta M, Segura $\mathrm{V}$, et al. Metastatic neuroblastoma in infants: are survival rates excellent only within the stringent framework of clinical trials? Clinical \& Translational Oncology. 2017; 19: 76-83.

[72] Mangus RS, Bush WJ, Miller C, Kubal CA. Severe Sarcopenia and Increased Fat Stores in Pediatric Patients with Liver, Kidney, or Intestine Failure. Journal of Pediatric Gastroenterology \& Nutrition. 2017; 65: 579-583.

[73] Lurz E, Patel H, Frimpong RG, Ricciuto A, Kehar M, Wales PW, et al. Sarcopenia in Children with End-Stage Liver Disease. 
Journal of Pediatric Gastroenterology and Nutrition. 2018; 66: 222-226.

[74] Suzuki D, Kobayashi R, Sano H, Hori D, Kobayashi K. Sarcopenia after induction therapy in childhood acute lymphoblastic leukemia: its clinical significance. International Journal of Hematology. 2018; 107: 486-489.

[75] López JJ, Cooper JN, Albert B, Adler B, King D, Minneci PC. Sarcopenia in children with perforated appendicitis. Journal of Surgical Research. 2017; 220: 1-5.

[76] Zhang H, Tao Y, Wang Z, Lu J. Evaluation of nutritional status and prognostic impact assessed by the prognostic nutritional index in children with chronic kidney disease. Medicine. 2019; 98: e16713.

[77] Kawakubo N, Kinoshita Y, Souzaki R, Koga Y, Oba U, Ohga S, et al. The Influence of Sarcopenia on High-Risk Neuroblastoma. Journal of Surgical Research. 2019; 236: 101-105.

[78] Lurz E, Patel H, Lebovic G, Quammie C, Woolfson JP, Perez $\mathrm{M}$, et al. Paediatric reference values for total psoas muscle area. Journal of Cachexia, Sarcopenia and Muscle. 2020; 11: 405414

[79] Morrell GR, Ikizler TA, Chen X, Heilbrun ME, Wei G, Boucher $\mathrm{R}$, et al. Psoas Muscle Cross-sectional Area as a Measure of whole-body Lean Muscle Mass in Maintenance Hemodialysis Patients. Journal of Renal Nutrition. 2016; 26: 258-264.

[80] Triarico S, Rinninella E, Mele MC, Cintoni M, Attinà G, Ruggiero A. Prognostic impact of sarcopenia in children with cancer: a focus on the psoas muscle area (PMA) imaging in the clinical practice. European Journal of Clinical Nutrition. 2021. (in press)

[81] Rinninella E, Cintoni M, Raoul P, Pozzo C, Strippoli A, Ponziani FR, et al. Skeletal Muscle Loss during Multikinase Inhibitors Therapy: Molecular Pathways, Clinical Implications, and $\mathrm{Nu}-$ tritional Challenges. Nutrients. 2020; 12: 3101.

[82] Rinninella E, Fagotti A, Cintoni M, Raoul P, Scaletta G, Scam- bia $\mathrm{G}$, et al. Skeletal muscle mass as a prognostic indicator of outcomes in ovarian cancer: a systematic review and metaanalysis. International Journal of Gynecologic Cancer. 2020; 30: 654-663.

[83] Oñate-Ocaña LF, Aiello-Crocifoglio V, Gallardo-Rincón D, Herrera-Goepfert R, Brom-Valladares R, Carrillo JF, et al. Serum albumin as a significant prognostic factor for patients with gastric carcinoma. Annals of Surgical Oncology. 2007; 14: 381-389.

[84] Rinninella E, Cintoni M, Raoul P, Pozzo C, Strippoli A, Bria $\mathrm{E}$, et al. Effects of nutritional interventions on nutritional status in patients with gastric cancer: a systematic review and metaanalysis of randomized controlled trials. Clinical Nutrition ESPEN. 2020; 38: 28-42.

[85] Delmore G. Assessment of nutritional status in cancer patients: widely neglected? Supportive Care in Cancer. 1997; 5: 376 380 .

[86] Hazzard E, Walton K, McMahon A, Milosavljevic M, Tapsell LC. Healthcare professionals' perspectives on the role of dietitians within multidisciplinary head and neck cancer teams: a qualitative multi-site study. Nutrition \& Dietetics. 2021; 78: 506-515.

[87] Ruggiero A, Cefalo MG, Coccia P, Mastrangelo S, Maurizi P, Riccardi R. The role of diet on the clinical pharmacology of oral antineoplastic agents. European Journal of Clinical Pharmacology. 2012; 68: 115-122.

[88] Morita M, Kudo K, Shima H, Tanuma N. Dietary intervention as a therapeutic for cancer. Cancer Science. 2021; 112: 498-504.

[89] Dokal K, Asmar N, Shergill-Bonner R, Mutalib M. Nutrition Evaluation Screening Tool: An Easy to Use Screening Tool for Hospitalised Children. Pediatric Gastroenterology, Hepatology \& Nutrition. 2021; 24: 90-99. 